Greybody factors for rotating black holes on codimension-2 branes

This article has been downloaded from IOPscience. Please scroll down to see the full text article.

JHEP03(2008)019

(http://iopscience.iop.org/1126-6708/2008/03/019)

The Table of Contents and more related content is available

Download details:

IP Address: 140.112.113.225

The article was downloaded on 17/07/2009 at 08:49

Please note that terms and conditions apply. 


\title{
Greybody factors for rotating black holes on codimension- 2 branes
}

\author{
Songbai Chen, ${ }^{a b}$ Bin Wang ${ }^{a}$ and Rukeng Su ${ }^{a c}$ \\ ${ }^{a}$ Department of Physics, Fudan University, \\ 200433 Shanghai, P.R. China \\ ${ }^{b}$ Institute of Physics and Department of Physics, Hunan Normal University, \\ Changsha, 410081 Hunan \\ ${ }^{c}$ China Center of Advanced Science and Technology (World Laboratory), \\ P.B.Box 8730, 100080 Beijing, P.R. China \\ E-mail: csb3752@163.com, wangb@fudan.edu.cn, rksu@fudan.ac.cn
}

\section{W.-Y. Pauchy Hwang}

Department of Physics, National Taiwan University, 106 Taipei, P.R. China

E-mail: wyhwang@phys.ntu.edu.tw

\begin{abstract}
We study the absorption probability and Hawking radiation of the scalar field in the rotating black holes on codimension-2 branes. We find that finite brane tension modifies the standard results in Hawking radiation if compared with the case when brane tension is completely negligible. We observe that the rotation of the black hole brings richer physics. Nonzero angular momentum triggers the super-radiance which becomes stronger when the angular momentum increases. We also find that rotations along different angles influence the result in absorption probability and Hawking radiation. Compared with the black hole rotating orthogonal to the brane, in the background that black hole spins on the brane, its angular momentum brings less super-radiance effect and the brane tension increases the range of frequency to accommodate super-radiance. These information can help us know more about the rotating codimension-2 black holes.
\end{abstract}

KEYwords: Black Holes in String Theory, Black Holes. 


\section{Contents}

1. Introduction 1

2. The master equation in rotating black holes on codimension-2 branes 3

3. Greybody factor in the low-energy regime 5

4. The absorption probability and Hawking radiation in the rotating black holes on codimension-2 branes 8

5. Conclusions and discussions 12

\section{Introduction}

In braneworld scenarios it is known that large extra dimensions can lower the fundamental scale of gravity down to the order of $\mathrm{TeV}$. This brings the possibility of creating microscopic black holes in high energy experiments such as the forthcoming Large Hadron Collider (LHC) [1] (for a recent review on this topic and a complete list of references please refer to [2]). After their creation, these microscopic black holes will decay quickly through the emission of Hawking radiation. Hawking radiation serves as the chief arena for studying the quantum gravity and disclosing the signature of extra dimensions [3]- 14]. Other attempts on detecting the extra dimension have been investigated in the perturbations around braneworld black holes [15]-19].

Most examinations for the extra dimension through Hawking radiations and the wave dynamics are concentrated on braneworld black holes with zero brane tension. However the nonzero tension on the brane is not trivial, since it can curve the brane as well as the bulk. In general it is very hard to obtain exact solutions of higher-dimensional Einstein equations describing black holes on the brane with tension. Recently, a metric describing a black hole located on a three-brane with finite tension, embedded in locally flat sixdimensional spacetime was constructed in 20]. Hawking radiation [10] and perturbations around this black hole [17, 19 have been studied subsequently, where modifications due to the finite brane tension and imprints of extra dimensions have been examined.

Further progress has been made by generalizing the Schwarzschild like solution of [20] to rotating black holes on a codimension-2 brane [21]. Including the rotation will bring richer physics. In Hawking radiation the amplifications in various properties due to the rotation have been shown in 12 14 where the brane tension was zero. It is of great interest to examine the evaporation of the rotating black holes on codimension-2 brane and investigate the physics brought by the rotation and the brane tension. 
The general metric of a small rotating black holes on a codimension-2 brane reads [21]

$$
\begin{aligned}
d s^{2}= & -\left(1-\frac{\mu r}{\Pi F}\right) d t^{2}+\frac{2 \mu r}{\Pi F}\left(a_{1} \mu_{1}^{2} d \phi_{1}+b a_{2} \mu_{2}^{2} d \phi_{2}\right) d t+\frac{F}{1-\frac{\mu r}{\Pi}} d r^{2} \\
& +r^{2}\left(d \alpha^{2}+d \mu_{1}^{2}+d \mu_{2}^{2}+\mu_{1}^{2} d \phi_{1}^{2}+b^{2} \mu_{2}^{2} d \phi_{2}^{2}\right) \\
& +a_{1}^{2}\left[d \mu_{1}^{2}+\mu_{1}^{2}\left(1+\frac{\mu r}{\Pi F} \mu_{1}^{2}\right) d \phi_{1}^{2}\right]+a_{2}^{2}\left[d \mu_{2}^{2}+b^{2} \mu_{2}^{2}\left(1+\frac{\mu r}{\Pi F} \mu_{2}^{2}\right) d \phi_{2}^{2}\right] \\
& +2 b a_{1} a_{2} \frac{\mu r}{\Pi F} \mu_{1}^{2} \mu_{2}^{2} d \phi_{1} d \phi_{2},
\end{aligned}
$$

with

$$
\Pi=\sum_{i=1}^{2}\left(r^{2}+a_{i}^{2}\right), \quad F=\sum_{i=1}^{2} \frac{r^{2} \mu_{i}^{2}}{r^{2}+a_{i}^{2}}+\alpha^{2} .
$$

Here $a_{1}, a_{2}$ are two angular momentum parameters. The two direction cosines, $\mu_{i}$ and the coordinate $\alpha$ are constrained by

$$
\mu_{1}^{2}+\mu_{2}^{2}+\alpha^{2}=1
$$

The quantity $\mu=r_{s}^{3} / b$ is proportional to the mass of the black hole, where $r_{s}$ is the horizon radius of the usual six-dimensional Schwarzschild black hole. The parameter $b$ is related to the brane tension $\lambda$ by $b=1-\frac{\lambda}{2 \pi M_{*}^{4}}$ with $0<b \leq 1$, where $M_{*}$ is the fundamental mass scale of six-dimensional gravity.

There are two different choices of angles through which the hole can rotate. Choosing the rotation axis to be $\phi_{2}$, by setting $a_{1}=0, a_{2}=a$, and after doing rescale $\phi_{2} \rightarrow b \phi_{2}$ and substituting $\phi_{1}=\phi, \phi_{2}=\psi$, and

$$
\mu_{1}=\sin \theta, \quad \mu_{2}=\cos \theta \sin \chi, \quad \alpha=\cos \theta \cos \chi
$$

into equation (1.1), we can obtain the metric for the black hole spinning orthogonal to the brane with its angular momentum pointing along the brane

$$
\begin{aligned}
d s^{2}= & -\left(1-\frac{\mu}{r \rho^{2}}\right) d t^{2}+\frac{2 \mu a}{r \rho^{2}} \sin \theta^{2} d t d \phi+\frac{\rho^{2}}{\Delta} d r^{2}+\rho^{2} d \theta^{2} \\
& +\sin \theta^{2}\left(r^{2}+a^{2}+\frac{\mu a^{2} \sin \theta^{2}}{r \rho^{2}}\right) d \phi^{2}+r^{2} \cos \theta^{2}\left(d \chi^{2}+b^{2} \sin \chi^{2} d \psi^{2}\right),
\end{aligned}
$$

where $\rho^{2}=r^{2}+a^{2} \cos \theta^{2}$ and $\Delta=r^{2}+a^{2}-\mu / r$. The Hawking temperature and the angular velocity of the horizon can be expressed as

$$
T_{H}=\frac{3 r_{H}^{2}+a^{2}}{4 \pi r_{H}\left(r_{H}^{2}+a^{2}\right)},
$$

and

$$
\Omega_{H}=\frac{a}{\left(r_{H}^{2}+a^{2}\right)} .
$$


Due to the presence of $b$ the radius of horizon $r_{H}$ is larger than $r_{s}$ [21], the angular velocity at the horizon is smaller than that in the tensionless case.

Choosing $\phi_{1}$ as the rotation axis, the hole would be spinning on the brane with its angular momentum orthogonal to the brane, whose metric has the form

$$
\begin{aligned}
d s^{2}= & -\left(1-\frac{\mu}{r \rho^{2}}\right) d t^{2}+\frac{2 \mu a b}{r \rho^{2}} \sin \theta^{2} d t d \phi+\frac{\rho^{2}}{\Delta} d r^{2}+\rho^{2} d \theta^{2} \\
& +b^{2} \sin \theta^{2}\left(r^{2}+a^{2}+\frac{\mu a^{2} \sin \theta^{2}}{r \rho^{2}}\right) d \phi^{2}+r^{2} \cos \theta^{2}\left(d \chi^{2}+\sin \chi^{2} d \psi^{2}\right) .
\end{aligned}
$$

The Hawking temperature is the same as that in the previous case, but the angular velocity at the horizon becomes

$$
\Omega_{H}=\frac{a}{b\left(r_{H}^{2}+a^{2}\right)} .
$$

It is clear that this angular velocity is larger than that in the tensionless case because the radius of the black hole horizon $r_{H} \sim b^{-1 / 3} r_{s}$, and thus the denominator in equation (9) $b\left(r_{H}^{2}+a^{2}\right) \sim b^{1 / 3} r_{s}^{2}+b a^{2}<r_{s}^{2}+a^{2}$.

We will discuss the evaporation of scalar field in six-dimensional black holes pierced by a tense 3-brane rotating orthogonal to the brane and along the brane. We will calculate the corresponding absorption probabilities and luminosity of Hawking radiation analytically by employing the matching technique of combining the far-field and near-horizon parts of solutions in the low energy and angular momentum limit.

The organization of the paper is as follows: in the following section we will derive the master equation in rotating black holes on codimension- 2 branes. In section 3 we will present the solution in the low energy and low angular momentum limit by using the matching technique. In section 4 , we derive the absorption probability and the luminosity of Hawking radiation. Finally in the last section we present our conclusions.

\section{The master equation in rotating black holes on codimension-2 branes}

The equation of motion for a massless scalar particle propagating in the curved spacetime is described by

$$
\frac{1}{\sqrt{-g}} \partial_{\mu}\left(\sqrt{-g} g^{\mu \nu} \partial_{\nu}\right) \Phi(t, r, \theta, \phi, \chi, \psi)=0,
$$

where $\Phi(t, r, \theta, \phi, \chi, \psi)$ is the scalar field. Separating the scalar field into $\Phi(t, r, \theta, \phi, \chi, \psi)=$ $e^{-i \omega t+i m \phi+i \eta \psi} R(r) S(\theta) \Gamma(\chi)$, we can obtain the radial and angular equations for the metric (1.5)

$$
\begin{array}{r}
\frac{1}{r^{2}} \frac{d}{d r}\left[r^{2} \Delta \frac{d R(r)}{d r}\right]+\left[\frac{K_{1}^{2}}{\Delta}+2 a m \omega-a^{2} \omega^{2}-E_{l m j 1}-\frac{\Lambda(j, \eta) a^{2}}{r^{2}}\right] R(r)=0,(2,2 \\
\frac{1}{\sin \theta \cos \theta^{2}} \frac{d}{d \theta}\left[\sin \theta \cos \theta^{2} \frac{d}{d \theta}\right] S(\theta)+\left[\omega^{2} a^{2} \cos \theta^{2}-\frac{m^{2}}{\sin ^{2} \theta}-\frac{\Lambda(j, \eta)}{\cos ^{2} \theta}-E_{l m j 1}\right] S(\theta)=0, \\
\frac{1}{\sin \chi} \frac{d}{d \chi}\left[\sin \chi \frac{d}{d \chi} \Gamma(\chi)\right]+\left[\Lambda(j, \eta)-\frac{\eta^{2}}{b^{2} \sin ^{2} \chi}\right] \Gamma(\chi)=0,
\end{array}
$$


with

$$
K_{1}=\omega\left(r^{2}+a^{2}\right)-a m,
$$

and $\eta$ and $\Lambda(j, \eta)$ are angular eigenvalue.

Similarly, in the background metric (1.8) radial and angular equations can be expressed as

$$
\begin{array}{r}
\frac{1}{r^{2}} \frac{d}{d r}\left[r^{2} \Delta \frac{d R(r)}{d r}\right]+\left[\frac{K_{2}^{2}}{\Delta}+\frac{2 a m \omega}{b}-a^{2} \omega^{2}-E_{l m j 2}-\frac{j(j+1) a^{2}}{r^{2}}\right] R(r)=0, \\
\frac{1}{\sin \theta \cos \theta^{2}} \frac{d}{d \theta}\left[\sin \theta \cos \theta^{2} \frac{d}{d \theta}\right] S(\theta)+\left[\omega^{2} a^{2} \cos \theta^{2}-\frac{m^{2}}{b^{2} \sin ^{2} \theta}-\frac{j(j+1)}{\cos ^{2} \theta}-E_{l m j 2}\right] S(\theta)=0,
\end{array}
$$

with

$$
K_{2}=\omega\left(r^{2}+a^{2}\right)-\frac{a m}{b} .
$$

We limit ourselves to the case where $\omega a \ll 1$ and the deviation of the parameter $b$ from unity is very small which is physically justified for small brane tension. And then we can adopt the perturbation theory to calculate eigenvalues of angular equations (2.3), (2.4) and (2.7). This perturbation method was first used in [17] and was supported in [19]. As did in [17], we find the angular eigenvalue of eq. (2.4)

$$
\Lambda(j, \eta)=j(j+1)+\frac{\eta(2 j+1)\left(1-b^{2}\right)}{2 b^{2}} .
$$

The zeroth-order eigenfunctions of (2.3) and (2.7) can be given in terms of the Jacobi polynomials

$$
S_{0}(\theta)=(\sin \theta)^{|m|} \cos \theta^{|j|} P\left(\frac{l-j-m}{2}, j+\frac{1}{2}, m ; 1-2 \cos ^{2} \theta\right),
$$

and then angular eigenvalues of eqs. (2.3) and (2.7) can be expressed respectively

$$
\begin{aligned}
E_{l m j 1}= & l(l+3)+\frac{\eta(2 j+1)\left(1-b^{2}\right)}{2 b^{2}} \frac{[2(j+m)+1](2 j+3)+2(l-j-m)(l+j+m+3)}{(2 j+3)(2 j-1)} \\
& +a^{2} \omega^{2} \frac{2 j(j-1)+2 l(l+1)-2 m^{2}+4(l+j)+3}{(2 l+5)(2 l+1)} \\
E_{l m j 2}= & l(l+3)+\frac{m[2(j+m)+1]\left(1-b^{2}\right)}{2 b^{2}}+a^{2} \omega^{2} \frac{2 j(j-1)+2 l(l+1)-2 m^{2}+4(l+j)+3}{(2 l+5)(2 l+1)}
\end{aligned}
$$

where $l, m, j$ and $\eta$ are restricted by

$$
l \geq(j+|m|), \quad j \geq|\eta| \quad \text { and } \quad \frac{l-(j+m)}{2} \in\left\{0, \mathbb{Z}^{+}\right\} .
$$

In the limit $b \rightarrow 1$, the eigenvalue $E_{l m j 1}$ is identical to $E_{l m j 2}$ and returns to that in the six dimensional rotating black hole spacetime without brane tension. 


\section{Greybody factor in the low-energy regime}

Now we provide an analytic solution of the radial equation using the matching technique. We first derive the solution in the near horizon regime, then derive the solution in the far field limit. Finally we stretch and match the two solutions in an intermediate region. In this way we can construct the analytic expression in the low energy and low angular momentum approximation for the radial part of the field valid throughout the entire spacetime. This analytic approximation has been employed in [12-14].

Let us first focus on the near-horizon regime. In order to translate radial equations (2.2) and (2.6) into the form of the known differential equation, we make the following change of the variable

$$
r \rightarrow f=\frac{\Delta}{r^{2}+a^{2}} \Rightarrow \frac{d f}{d r}=(1-f) r \frac{A}{r^{2}+a^{2}},
$$

where $A=3+a^{2} / r^{2}$. Then radial equations (2.2) and (2.6) can be rewritten in a unified form

$$
f(1-f) \frac{d^{2} R(f)}{d f^{2}}+\left(1-D_{*} f\right) \frac{d R(f)}{d f}+\left[\frac{K_{*, i}^{2}}{A\left(r_{H}\right)^{2}(1-f) f}-\frac{\left(E_{l m j i}-P_{i}\right)\left(r_{H}^{2}+a^{2}\right)}{r_{H}^{2} A\left(r_{H}\right)^{2}(1-f)}\right] R(f)=0,(3
$$

with $i=1,2$ corresponding to new radial equations in the background of (1.5) and (1.8) respectively.

$$
D_{*}=1-\frac{4 a^{2} r_{H}^{2}}{\left(3 r_{H}^{2}+a^{2}\right)^{2}}
$$

and

$$
\begin{array}{ll}
K_{*, 1}=\omega\left(r_{H}+\frac{a^{2}}{r_{H}}\right)-\frac{a m}{r_{H}}, & P_{1}=2 a m \omega-a^{2} \omega^{2}-\frac{\Lambda(j, \eta) a^{2}}{r_{H}^{2}}, \\
K_{*, 2}=\omega\left(r_{H}+\frac{a^{2}}{r_{H}}\right)-\frac{a m}{b r_{H}}, & P_{2}=\frac{2 a m \omega}{b}-a^{2} \omega^{2}-\frac{j(j+1) a^{2}}{r_{H}^{2}} .
\end{array}
$$

for black holes on codimension-2 branes rotating orthogonal to the brane or on the brane, respectively. Making the field redefinition $R(f)=f^{\alpha}(1-f)^{\beta} F(f)$, we can rewrite equation (3.2) in the form of a hypergeometric equation

$$
f(1-f) \frac{d^{2} F(f)}{d f^{2}}+\left[c-\left(1+\tilde{a}_{i}+\tilde{b}_{i}\right) f\right] \frac{d F(f)}{d f}-\tilde{a}_{i} \tilde{b}_{i} F(f)=0,
$$

where

$$
\tilde{a}_{i}=\alpha_{i}+\beta_{i}+D_{*}-1, \quad \tilde{b}_{i}=\alpha_{i}+\beta_{i}, \quad c_{i}=1+2 \alpha_{i} .
$$

Due to the constraint from the coefficient of $F(f)$, the power coefficients $\alpha_{i}$ and $\beta_{i}$ must satisfy the second-order algebraic equations

$$
\alpha_{i}^{2}+\frac{K_{*, i}^{2}}{A\left(r_{H}\right)^{2}}=0
$$


and

$$
\beta_{i}^{2}+\beta_{i}\left(D_{*}-2\right)+\frac{K_{*, i}^{2}}{A\left(r_{H}\right)^{2}}-\frac{\left(E_{l m j i}-P_{i}\right)\left(r_{H}^{2}+a^{2}\right)}{r_{H}^{2} A\left(r_{H}\right)^{2}}=0 .
$$

Solving these two equations, we obtain solutions for parameters $\alpha$ and $\beta$

$$
\begin{aligned}
& \alpha_{i \pm}= \pm \frac{i K_{*, i}}{A\left(r_{H}\right)}, \\
& \beta_{i \pm}=\frac{1}{2}\left[\left(2-D_{*}\right) \pm \sqrt{\left(D_{*}-2\right)^{2}-\frac{4 K_{*, i}^{2}}{A\left(r_{H}\right)^{2}}+\frac{4\left(E_{l m j i}-P_{i}\right)\left(r_{H}^{2}+a^{2}\right)}{r_{H}^{2} A\left(r_{H}\right)^{2}}}\right] .
\end{aligned}
$$

Thus, the general solution of master equations (2.2) and (2.6) near the horizon can be expressed as

$R_{i N H}(f)=A_{i-} f^{\alpha_{i}}(1-f)^{\beta_{i}} F\left(\tilde{a_{i}}, \tilde{b_{i}}, c_{i} ; f\right)+A_{i+} f^{-\alpha}(1-f)^{\beta} F\left(\tilde{a_{i}}-c_{i}+1, \tilde{b_{i}}-c_{i}+1,2-c_{i} ; f\right)$,

where $A_{i \pm}$ are arbitrary constants. Near the horizon, $r \rightarrow r_{H}$, and $f \rightarrow 0$, the solution (3.12) can be reduced to

$$
R_{i N H}(f)=A_{i-} f^{ \pm i K_{*, i} / A\left(r_{H}\right)}+A_{i+} f^{\mp i K_{*, i} / A\left(r_{H}\right)}=A_{i-} e^{ \pm i k_{i} y}+A_{i+} e^{\mp i k_{i} y},
$$

with

$$
k_{1}=\omega-\frac{a m}{r_{H}^{2}+a^{2}}, \quad k_{2}=\omega-\frac{a m}{b\left(r_{H}^{2}+a^{2}\right)},
$$

for different rotating angles. Here $y$ is the tortoise-like coordinate, which can be expressed as

$$
y=\frac{\left(r_{H}^{2}+a^{2}\right) \ln f}{r_{H} A\left(r_{H}\right)} .
$$

According to the boundary condition that no outgoing mode exists near the horizon, we choose $\alpha_{i}=\alpha_{i-}$ and $A_{i+}=0$. Thus the asymptotic near horizon solution has the form

$$
R_{i N H}(f)=A_{i-} f^{\alpha_{i}}(1-f)^{\beta_{i}} F\left(\tilde{a_{i}}, \tilde{b_{i}}, c_{i} ; f\right) .
$$

Moreover, the boundary condition also demands that near the horizon the hypergeometric function $F\left(\tilde{a_{i}}, \tilde{b_{i}}, c_{i} ; f\right)$ must be convergent, i.e. $\operatorname{Re}\left(c_{i}-\tilde{a_{i}}-\tilde{b_{i}}\right)>0$, which implies that we must choose $\beta_{i}=\beta_{i-}$.

Now, let us turn to the far field region, where equations (2.2) and (2.6) take the form

$$
\frac{d^{2} R_{F F}(r)}{d r^{2}}+\frac{4}{r} \frac{d R_{F F}(r)}{d r}+\left[\omega^{2}-\frac{E_{l m j i}+a^{2} \omega^{2}}{r^{2}}\right] R_{F F}(r)=0 .
$$

Obviously, this is a Bessel equation. General solutions of radial master equations (2.2) and (2.6) in the far field region can be expressed as

$$
R_{i F F}(r)=\frac{1}{\sqrt{r}}\left[B_{i 1} J_{\nu_{i}}(\omega r)+B_{i 2} Y_{\nu_{i}}(\omega r)\right]
$$


where $J_{\nu_{i}}(\omega r)$ and $Y_{\nu_{i}}(\omega r)$ are the first and second kind Bessel functions, $\nu_{i}=$ $\sqrt{E_{l m j i}+a^{2} \omega^{2}+9 / 4} . B_{i 1}$ and $B_{i 2}$ are integration constants.

In order to match the near horizon and far field solutions in the intermediate zone, we must stretch the near horizon solution to the large value of the radial coordinate. As in refs. [12-14], at first we change the argument of the hypergeometric function of the near-horizon solution from $f$ to $1-f$ by using the relation

$$
\begin{aligned}
R_{i N H}(f)= & A_{-} f^{\alpha_{i}}(1-f)^{\beta_{i}}\left[\frac{\Gamma(c-i) \Gamma\left(c_{-} \tilde{a_{i}}-\tilde{b_{i}}\right)}{\Gamma\left(c_{i}-\tilde{a_{i}}\right) \Gamma\left(c_{i}-\tilde{b_{i}}\right)} F\left(\tilde{a_{i}}, \tilde{b_{i}}, \tilde{a_{i}}+\tilde{b_{i}}-c_{i}+1 ; 1-f\right)\right. \\
& \left.+(1-f)^{c_{i}-\tilde{a_{i}}-\tilde{b_{i}}} \frac{\Gamma\left(c_{i}\right) \Gamma\left(\tilde{a_{i}}+\tilde{b_{i}}-c_{i}\right)}{\Gamma\left(\tilde{a_{i}}\right) \Gamma\left(\tilde{b_{i}}\right)} F\left(c_{i}-\tilde{a_{i}}, c_{i}-\tilde{b_{i}}, c_{i}-\tilde{a_{i}}-\tilde{b_{i}}+1 ; 1-f\right)\right] .(3.1
\end{aligned}
$$

In the limit $r \gg r_{H}$, the function $(1-f)$ can be approximated by

$$
1-f=\frac{\mu}{r} \frac{1}{r^{2}+a^{2}} \sim \frac{r_{H}\left(r_{H}^{2}+a^{2}\right)}{r^{3}}
$$

and the near-horizon solution $(\sqrt{3.19})$ can be simplified further as

$$
R_{i N H}(r) \simeq A_{i 1} r^{-3 \beta_{i}}+A_{i 2} r^{3\left(\beta_{i}+D_{*}-2\right)}
$$

with

$$
\begin{aligned}
& A_{i 1}=A_{i-} \frac{\Gamma\left(c_{i}\right) \Gamma\left(c_{i}-\tilde{a_{i}}-\tilde{b_{i}}\right)}{\Gamma\left(c_{i}-\tilde{a_{i}}\right) \Gamma\left(c_{i}-\tilde{b_{i}}\right)}\left[r_{H}\left(r_{H}^{2}+a^{2}\right)\right]^{\beta_{i}}, \\
& A_{i 2}=A_{i-} \frac{\Gamma\left(c_{i}\right) \Gamma\left(\tilde{a_{i}}+\tilde{b_{i}}-c_{i}\right)}{\Gamma\left(\tilde{a_{i}}\right) \Gamma\left(\tilde{b_{i}}\right)}\left[r_{H}\left(r_{H}^{2}+a^{2}\right)\right]^{-\left(\beta+D_{*}-2\right)} .
\end{aligned}
$$

In the limit $r \rightarrow 0, R_{i F F}(r)$ in equation (3.18) becomes

$$
R_{i F F}(r) \simeq \frac{B_{i 1}\left(\frac{\omega r}{2}\right)^{\nu_{i}}}{\sqrt{r} \Gamma\left(\nu_{i}+1\right)}-\frac{B_{i 2} \Gamma\left(\nu_{i}\right)}{\pi \sqrt{r}\left(\frac{\omega r}{2}\right)^{\nu_{i}}} .
$$

And then comparing it with equation (3.21), we will obtain two relations between $A_{i 1}$ and $B_{i 1}, B_{i 2}$ in the limit $\omega r_{H} \ll 1$. Then making use of equations (3.22) and (3.23) and removing $A_{i-}$, we find that the constraint for $B_{i 1}, B_{i 2}$ is given by

$$
\begin{aligned}
B_{i} \equiv \frac{B_{i 1}}{B_{i 2}}= & -\frac{1}{\pi}\left[\frac{2}{\omega r_{H}^{1 / 3}\left(r_{H}^{2}+a^{2}\right)^{1 / 3}}\right]^{2 l+3} \sqrt{E_{l m j i}+a^{2} \omega^{2}+9 / 4} \\
& \times \frac{\Gamma^{2}\left(\sqrt{E_{l m j}+a^{2} \omega^{2}+9 / 4}\right) \Gamma\left(c_{i}-\tilde{a}_{i}-\tilde{b_{i}}\right) \Gamma\left(\tilde{a_{i}}\right) \Gamma\left(\tilde{b_{i}}\right)}{\Gamma\left(\tilde{a}_{i}+\tilde{b}_{i}-c_{i}\right) \Gamma\left(c_{i}-\tilde{a_{i}}\right) \Gamma\left(c_{i}-\tilde{b}_{i}\right)} .
\end{aligned}
$$

In the asymptotic region $r \rightarrow \infty$, the solution in the far field can be expressed as

$$
R_{i F F}(r) \simeq \frac{B_{i 1}+i B_{i 2}}{2 \sqrt{2 \pi \omega} r} e^{-i \omega r}+\frac{B_{i 1}-i B_{i 2}}{2 \sqrt{2 \pi \omega} r} e^{i \omega r}=A_{i \text { in }}^{(\infty)} \frac{e^{-i \omega r}}{r}+A_{i \text { out }}^{(\infty)} \frac{e^{i \omega r}}{r}
$$


The absorption probability can be calculated by

$$
\left|\mathcal{A}_{l m j i}\right|^{2}=1-\left|\frac{A_{i \text { out }}^{(\infty)}}{A_{i \text { in }}^{(\infty)}}\right|^{2}=1-\left|\frac{B_{i}-i}{B_{i}+i}\right|^{2}=\frac{2 i\left(B_{i}^{*}-B_{i}\right)}{B_{i} B_{i}^{*}+i\left(B_{i}^{*}-B_{i}\right)+1} .
$$

Combining the above result and the expression $B_{i}$ given in equation (3.25), we can examine properties of absorption probability for the massless scalar field in two kinds of classical rotating black holes on codimension-2 branes in the low-energy and low-angular momentum limit.

\section{The absorption probability and Hawking radiation in the rotating black holes on codimension-2 branes}

With solutions obtained above, we are now in a position to compute the absorption probability and discuss Hawking radiation of black holes on codimension-2 branes spinning orthogonal to the brane and spinning on the brane, respectively. Recently numerical study on the emission of scalar fields into the bulk from a six-dimensional tensional black hole rotating orthogonal to the brane was also proposed in [22].

In figure 1, we examine the influence of the brane tension on the absorption probability. We plot the absorption probability for the first partial waves $(l=0, m=0, j=0)$ by fixing $a=0.4$. It is clearly shown that the absorption probability decreases with the increase of $b$ (decrease of the brane tension). In figure 2 we fix $b$ (with constant brane tension) and exhibit the dependence of the absorption probability on the angular momentum. It shows that with the increase of the angular momentum, the absorption probability decreases. The main reason is that in the low-energy limit the absorption probability $\mid \mathcal{A}(l=0, m=$ $0, j=0)\left.\right|^{2} \sim \omega^{4} r_{H}^{4}$. When parameters $a$ and $b$ increase, the radius of the black hole event horizon decreases. The dependence of the absorption on the brane tension and angular momentum of the black hole does not differ much for the six-dimensional tensional black holes rotating along different angles.

Figure 2 shows the dependence of the absorption probability on the angular index. We see the suppression of $|\mathcal{A}|^{2}$ as the values of the angular index increase. This phenomenon explains that the first partial wave dominates over all others, which has also been observed in black hole cases when there is no brane tension 12, 13]. Moreover, we also observe that the absorption probability in the black hole (1.5) depends on the angular index $\eta$. With the increase of the index $\eta$, the absorption probability decreases.

In figure 3 , we find that for positive $m$, in some ranges of frequency $\omega$, the absorption probability can be negative, which presents us the super-radiance. This is the property brought by the rotation as also disclosed in [12, 13]. Here we observed that the brane tension also influences the super-radiation. For the black hole spinning orthogonal to the brane, the range of $\omega$ for the super-radiance to occur increases with the increase of $b$ (decrease of the brane tension). But for the black hole spinning on the brane, with the decrease of the brane tension, the range of $\omega$ for the super-radiance to happen decreases. The physical reason behind this phenomenon can be understood as follows. 

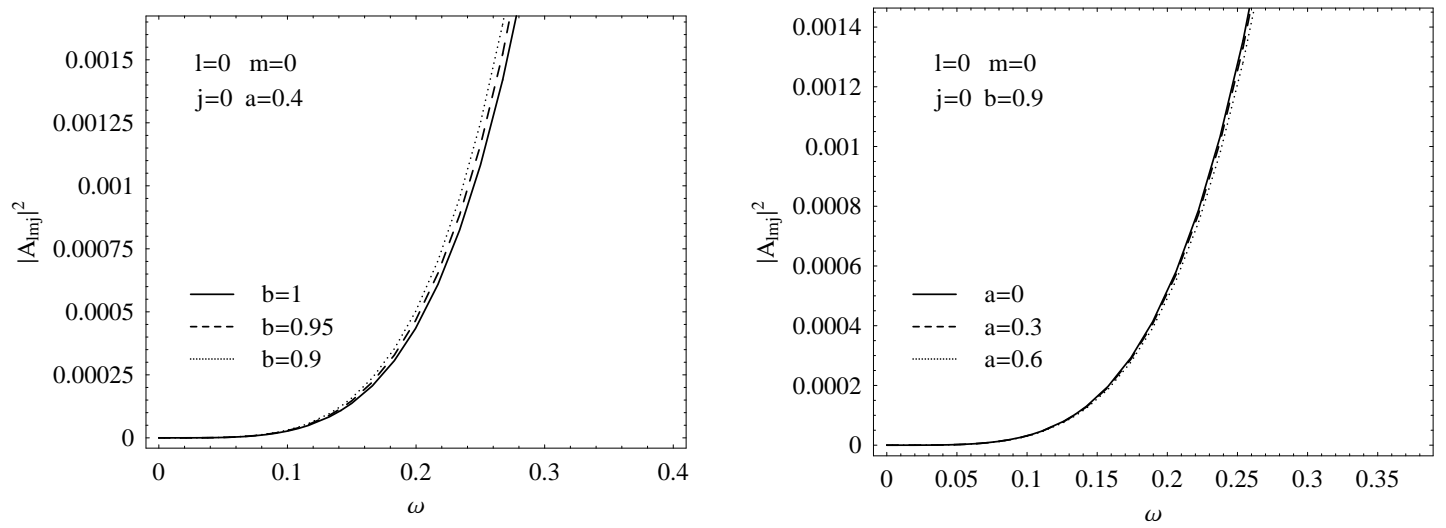

Figure 1: Absorption probability $|\mathcal{A}|^{2}$ of scalar particles propagating in the rotating black holes on codimension-2 branes, for different $b$ and $a$, respectively, when $l=0, j=0, m=0$. Here $r_{s}=1$.

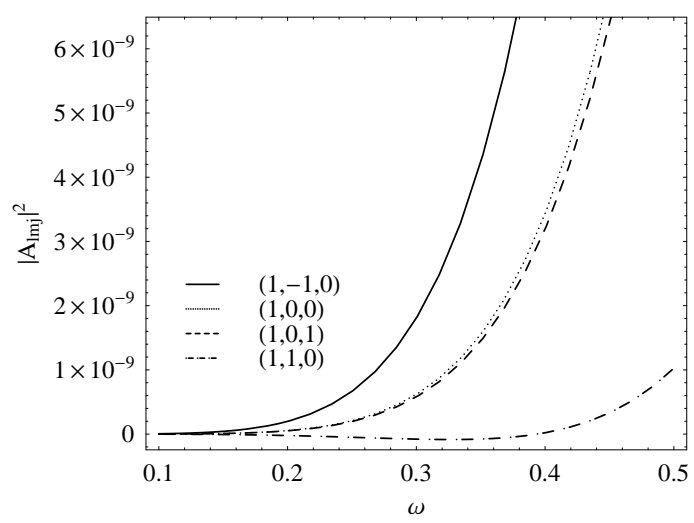

Figure 2: Absorption probability $|\mathcal{A}|^{2}$ of scalar particles propagating in the rotating black holes on codimension-2 branes for different combinations of $(l, m, j)$, where we fixed $a=0.4, b=0.9$ and $r_{s}=1$.

As in [12, 13], in the low energy limit $B B * \gg i(B *-B) \gg 1$, we can simplify our (3.27) to the form

$$
\begin{aligned}
\left|\mathcal{A}_{l m j i}\right|^{2} & =2 i\left(\frac{1}{B}-\frac{1}{B *}\right) \\
& =\frac{4 \pi\left[\omega r_{H}^{1 / 3}\left(r_{H}^{2}+a^{2}\right)^{1 / 3} / 2\right]^{2 l+3} K_{*, i} \Gamma^{2}\left(2 \beta+D_{*}-2\right) \Gamma^{2}(1-\beta)\left(2-D_{*}-2 \beta\right)}{A\left(r_{H}\right) \sqrt{E_{l m j i}+9 / 4} \Gamma^{2}\left(\sqrt{E_{l m j i}+9 / 4}\right) \Gamma^{2}\left(\beta+D_{*}-1\right) \sin ^{2}\left(\pi\left(\beta+D_{*}\right)\right)} .
\end{aligned}
$$

From (3.11) we learnt that the quantity $2-D_{*}-2 \beta$ is always positive. The possibility to make $\left|\mathcal{A}_{l m j i}\right|^{2}<0$ is $K_{*, i}<0$, which leads

$$
0 \leq \omega_{1} \leq \omega_{c, 1}=\frac{a m}{r_{H}^{2}+a^{2}},
$$



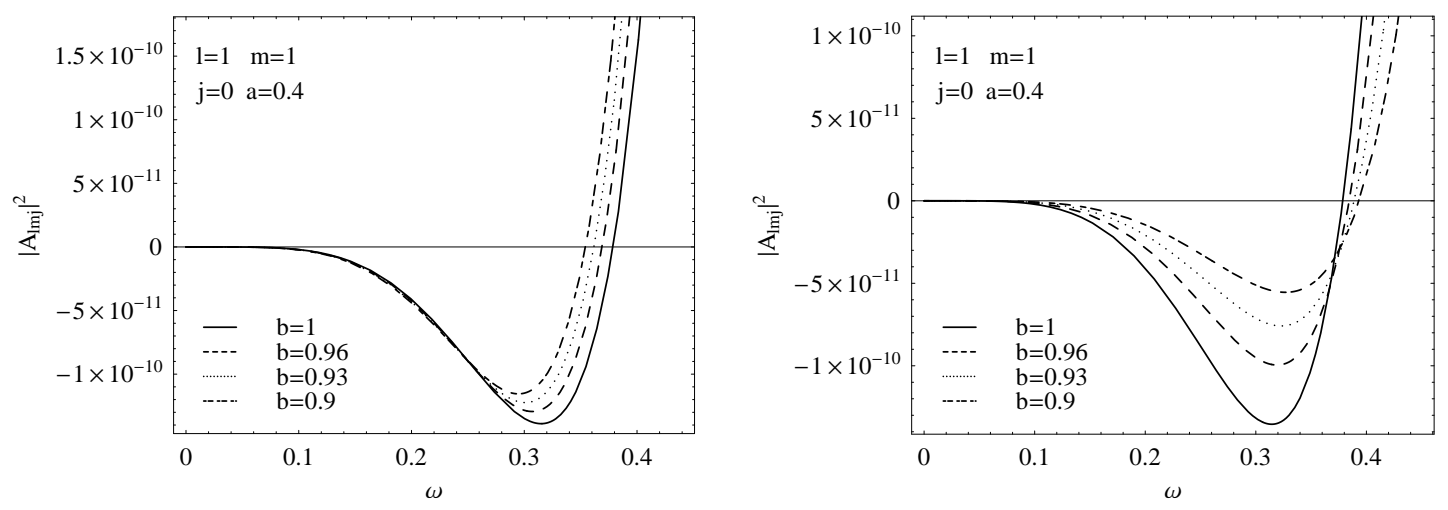

Figure 3: Absorption probability $|\mathcal{A}|^{2}$ of scalar particles propagating in the rotating black holes (the left for the metric (1.5) and the right for the metric (1.8)) on codimension-2 branes, for fixed $a=0.4, l=1, m=1, j=0$, and different $b$. Here $r_{s}=1$.
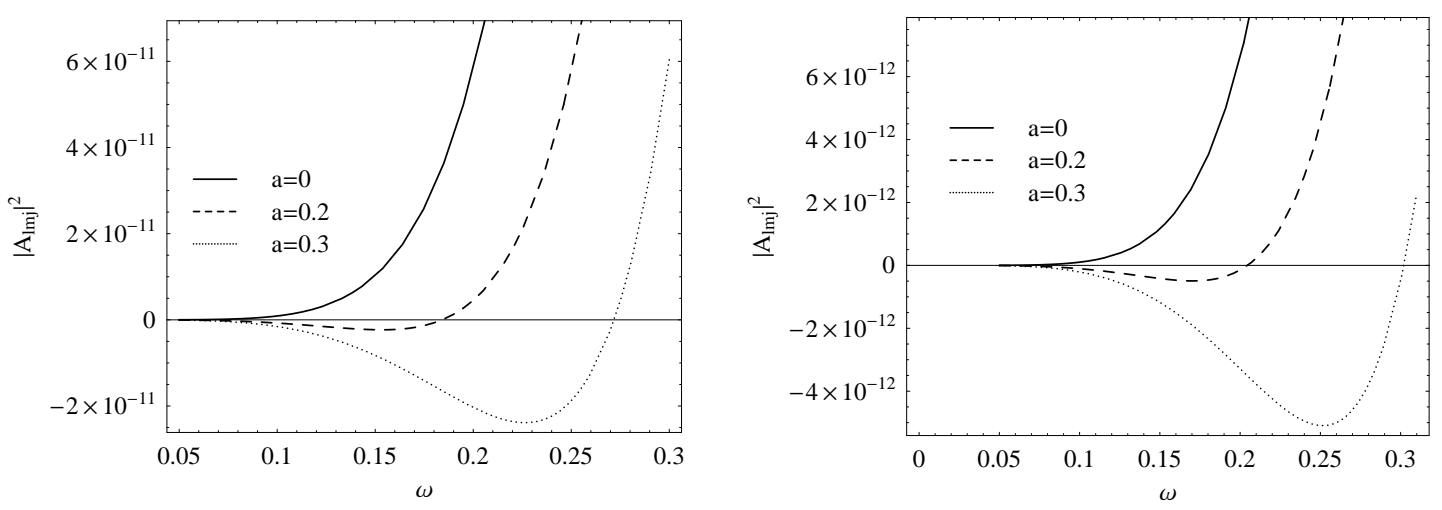

Figure 4: The super-radiance for the mode $l=1, m=1, j=0$ in the metrics (1.5) (the left) and (1.8) (the right) for fixed $b=0.9$ and different $a$. Here $r_{s}=1$.

for the black hole spinning orthogonal to the brane and

$$
0 \leq \omega_{2} \leq \omega_{c, 2}=\frac{a m}{b\left(r_{H}^{2}+a^{2}\right)}
$$

for the hole spinning on the brane. Since $r_{H} \sim r_{s} b^{-1 / 3}$, we have in the low angular momentum limit $\omega_{c, 1} \propto b^{2 / 3}$ and $\omega_{c, 2} \propto b^{-1 / 3}$, respectively. Thus with the increase of $b, \omega_{c, 1}$ increases while $\omega_{c, 2}$ decreases respectively for black holes rotating along different angles.

The dependence of the super-radiance on the angular momentum of the black hole is shown in figure 4. It is clear that the super-radiance occurs when $a>0$ and becomes stronger when $a$ increases. For the same angular momentum, it brings more super-radiance in the black hole background (1.5) than that in (1.8). For small $a$ the magnitude of superradiance is very small, which has little contribution to the luminosity of Hawking radiation. 

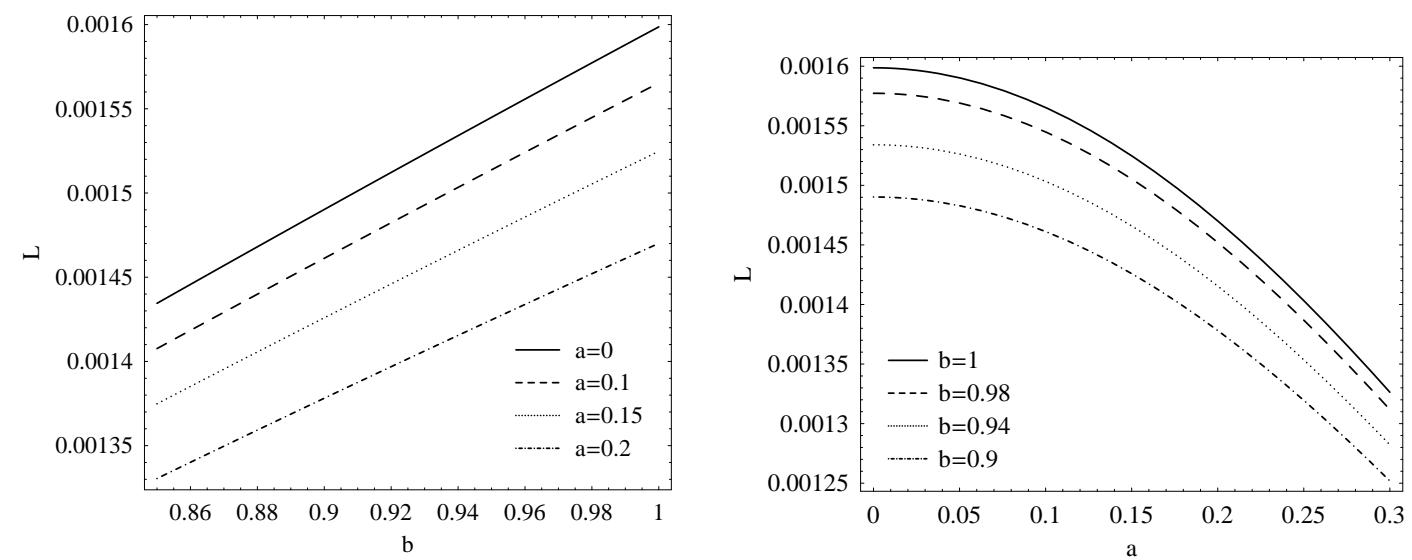

Figure 5: The luminosity of Hawking radiation $L$ of scalar particles propagating in the rotating black holes on codimension-2 branes $(l=1, m=1, j=0)$. The left shows the change of $L$ with $b$ for different $a$ and the right exhibits the change of $L$ with $a$ for different $b$. Here $r_{s}=1$.

Now let us turn to study the luminosity of Hawking radiation of black holes in backgrounds (1.5) and (1.8) for the mode $l=0, m=0, j=0$ which plays a dominant role in the greybody factor. For the first partial wave $(l=0, m=0, j=0)$, we have $K_{*, 1}=K_{*, 2}$, $P_{1}=P_{2}, \alpha_{1}=\alpha_{2}$ and $\beta_{1}=\beta_{2}$, thus in this case there is no difference in the absorption probability and Hawking radiation when black holes rotate along different angles. Performing an analysis similar to that in [12, 13], we can rewrite the the absorption probability (4.1) as

$$
\left|\mathcal{A}_{000}\right|^{2}=\frac{4 \omega^{4} r_{H}^{2}\left(r_{H}^{2}+a^{2}\right)\left(3 r_{H}^{2}+a^{2}\right)}{3\left(9 r_{H}^{2}+a^{2}\right)} \text {. }
$$

Combining it with equation (1.6), the luminosity of Hawking radiation is given by

$$
\begin{aligned}
L & =\int_{0}^{\infty} \frac{d \omega}{2 \pi}\left|\mathcal{A}_{000}\right|^{2} \frac{\omega}{e^{\omega / T_{H}}-1} \\
& =\frac{\left(3 r_{H}^{2}+a^{2}\right)^{7}}{48384 \pi r_{H}^{4}\left(r_{H}^{2}+a^{2}\right)^{5}\left(9 r_{H}^{2}+a^{2}\right)}=\frac{4 \pi^{5}}{63} G T_{H}^{6},
\end{aligned}
$$

where $G=\frac{4 r_{H}^{2}\left(r_{H}^{2}+a^{2}\right)\left(3 r_{H}^{2}+a^{2}\right)}{3\left(9 r_{H}^{2}+a^{2}\right)}$. In figure 5 , we show the dependence of the luminosity of Hawking radiation on parameter $b$ for fixed angular momentum parameters. It is clear that as $b$ increases, (decrease of the brane tension), $L$ increases. From the formula (4.5), we obtain that in the low rotating limit $L \sim r_{H}^{-2} \sim b^{2 / 3} r_{s}^{-2}$. This shows that the decrease of the brane tension enhances Hawking radiation. This effect can also be understood from the Hawking temperature. We have $\frac{d T_{H}}{d b}=\frac{3 r_{H}^{4}+a^{4}}{4 \pi b^{2} r_{H}^{2}\left(r_{H}^{2}+a^{2}\right)^{2}\left(3 r_{H}^{2}+a^{2}\right)}>0$ which indicates that the Hawking temperature increases with the increase of $b$, thus leading to the stronger Hawking radiation.

The luminosity of Hawking radiation depending on the angular momentum of the black hole is also studied. When $a$ increases, the luminosity of Hawking radiation $L$ decreases. This is because that the Hawking temperature decreases when the black hole 

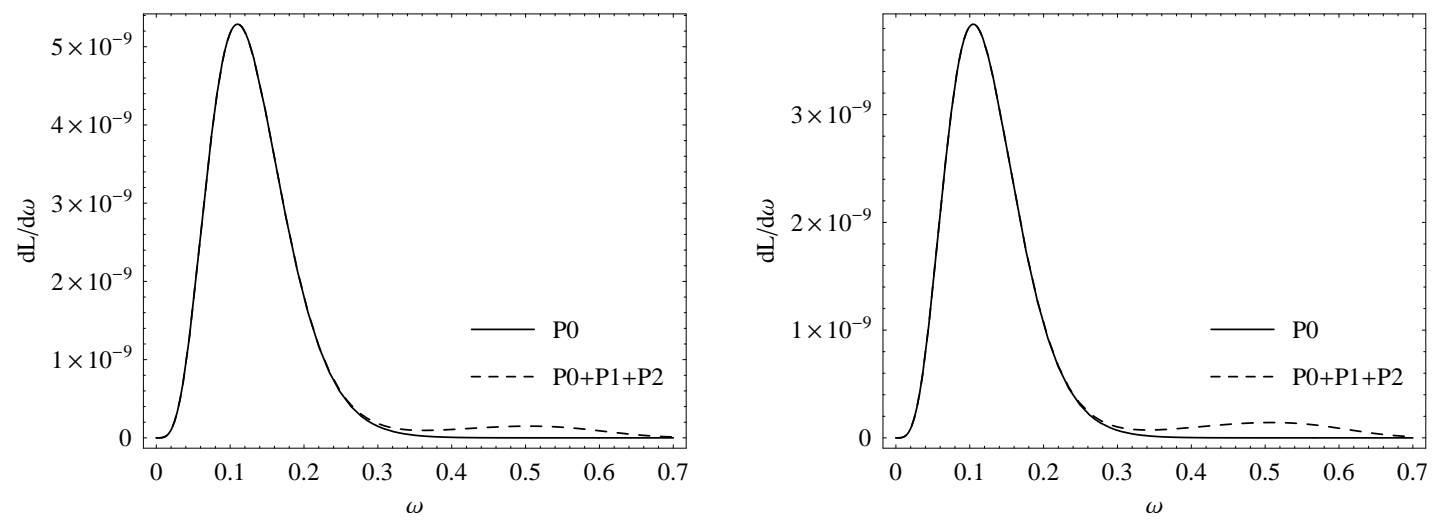

Figure 6: $P 0$ and $P 0+P 1+P 2$ in metric (1.5), the left for fixed $b=0.9$ and the right for fixed $b=0.95$, Here $a=1.4$ and $r_{s}=1$.

rotates faster, $\frac{d T_{H}}{d a}=-\frac{a\left(3 r_{H}^{2}-a^{2}\right)}{2 \pi r_{H}\left(3 r_{H}^{4}+4 a^{2} r_{H}^{2}+a^{4}\right)}<0$. We have also examined the emission rate

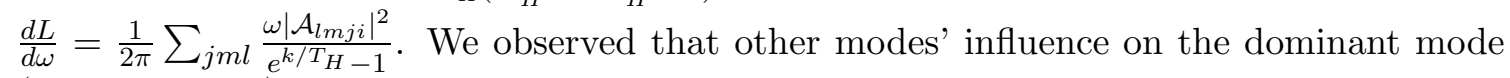
$(l=m=j=0)$ is negligible when $a$ is small. In figures 6 and 7 , we show the contribution of other mode on the dominant mode when $a=1.4$, which still satisfies the low energy limit $a \omega<1$. Variables $P 0, P 1, P 2$ in the figure are given by

$$
\begin{aligned}
& P 0=\frac{1}{2 \pi} \frac{\omega\left|\mathcal{A}_{000}\right|^{2}}{e^{k / T_{H}}-1}, \\
& P 1=\frac{1}{2 \pi}\left[\frac{\omega\left|\mathcal{A}_{110}\right|^{2}}{e^{k / T_{H}}-1}+\frac{\omega\left|\mathcal{A}_{1-10}\right|^{2}}{e^{k / T_{H}}-1}+\frac{\omega\left|\mathcal{A}_{100}\right|^{2}}{e^{k / T_{H}}-1}\right], \\
& P 2=\frac{1}{2 \pi}\left[\frac{\omega\left|\mathcal{A}_{220}\right|^{2}}{e^{k / T_{H}}-1}+\frac{\omega\left|\mathcal{A}_{210}\right|^{2}}{e^{k / T_{H}}-1}+\frac{\omega\left|\mathcal{A}_{200}\right|^{2}}{e^{k / T_{H}}-1}+\frac{\omega\left|\mathcal{A}_{2-10}\right|^{2}}{e^{k / T_{H}}-1}+\frac{\omega\left|\mathcal{A}_{2-20}\right|^{2}}{e^{k / T_{H}}-1}\right] .
\end{aligned}
$$

When $b$ increases, the modification becomes bigger. This is because that bigger $b$ leads stronger super-radiance. Comparing to the black hole background (1.5), the modification to the dominant mode's emission rate is even weaker in the background (1.8). This is consistent with the observation that for fixed $a$, there is not much super-radiance in the background (1.8) compared to that in (1.5). To our observation, in the low energy limit the enhancement of the emission rate due to the angular momentum and brane tension is not obvious. It is interesting to generalize our investigation to the intermediate and high energy and angular momentum situations to reexamine the emission rate of scalar field.

\section{Conclusions and discussions}

We have studied the absorption probability and Hawking radiation of scalar field in the background of six-dimensional black holes rotating orthogonal to a tensional brane or spinning on a tensional brane, respectively. Our results show that with the nonzero brane tension, properties of evaporations of scalar field are different from those of the rotating 

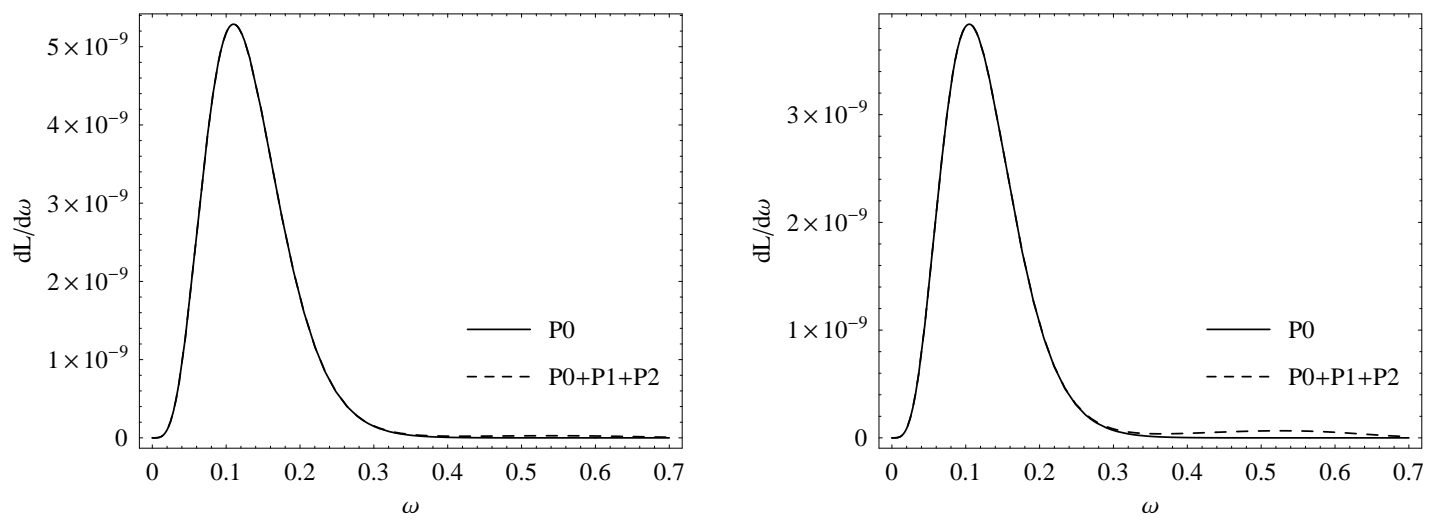

Figure 7: $P 0$ and $P 0+P 1+P 2$ in metric (1.8), the left for fixed $b=0.9$ and the right for fixed $b=0.95$. Here $a=1.4$ and $r_{s}=1$.

black holes where the brane tension is completely negligible. This could serve as signatures of extra dimensions in the future collider searches.

We have observed that the rotation of the black hole brings richer physics. Nonzero angular momentum triggers the super-radiance. But in our analytic analysis we see that the enhancement of the emission rate due to the angular momentum is weak in the low energy limit. The lowest mode still dominates over other modes in Hawking radiation and gets small modification in the low energy and angular momentum limits. It is of interest to generalize our investigation to the intermediate and high energy and angular momentum situations to reexamine the emission rate.

We have also compared the results for black holes rotating in different angles and found that when the black hole rotating on the tensional brane, the phenomenon of the superradiance caused by the angular momentum is not obvious as compared with the black hole rotating orthogonal to the brane. Less enhancement of the emission rate has been found for the hole spinning on the tensional brane. The effects due to the brane tension on the super-radiance also differ when black holes rotate along different angles. For the black hole rotating on the tensional brane, the nonzero brane tension allows bigger range of the frequency to accommodate the super-radiance.

In this paper we only considered the bulk scalar emission in the codimension-2 rotating black holes. It would be interesting to examine the emission of the scalar field on the brane and investigate the brane to bulk ratio of Hawking radiation. Recently for rotating black holes with zero tension on the brane, the ratio between the brane and bulk Hawking radiation has been studied in [14. Furthermore it would be of more interesting to study other fields emission, such as the gravitational field etc. Works in this direction will be reported in the future. 


\section{Acknowledgments}

This work was partially supported by NNSF of China, Shanghai Education Commission and Shanghai Science and Technology Commission. R. K. Su's work was partially supported by the National Basic Research Project of China. S. B. Chen's work was partially supported by the China Postdoctoral Science Foundation under Grant No. 20070410685, the Scientific Research Fund of Hunan Provincial Education Department Grant No. 07B043 and the National Basic Research Program of China under Grant No. 2003CB716300.

\section{References}

[1] S.B. Giddings and S.D. Thomas, High energy colliders as black hole factories: the end of short distance physics, Phys. Rev. D 65 (2002) 056010 hep-ph/0106219;

S. Dimopoulos and G.L. Landsberg, Black holes at the LHC, Phys. Rev. Lett. 87 (2001) 161602 hep-ph/0106295;

S. Dimopoulos and R. Emparan, String balls at the LHC and beyond, Phys. Lett. B 526 (2002) 393 hep-ph/0108060;

S. Hossenfelder, S. Hofmann, M. Bleicher and H. Stoecker, Quasi-stable black holes at LHC, Phys. Rev. D 66 (2002) 101502 hep-ph/0109085.

[2] P. Kanti, Black holes at the LHC, arXiv:0802.2218.

[3] P. Kanti, Reading the number of extra dimensions in the spectrum of Hawking radiation, hep-ph/0310162.

[4] C.M. Harris and P. Kanti, Hawking radiation from a $(4+n)$-dimensional black hole: exact results for the Schwarzschild phase, JHEP 10 (2003) 014 hep-ph/0309054;

P. Kanti, Black holes in theories with large extra dimensions: a review, Int. J. Mod. Phys. A 19 (2004) 4899 hep-ph/0402168.

[5] P.C. Argyres, S. Dimopoulos and J. March-Russell, Black holes and sub-millimeter dimensions, Phys. Lett. B 441 (1998) 96 hep-th/9808138;

T. Banks and W. Fischler, A model for high energy scattering in quantum gravity, hep-th/9906038;

R. Emparan, G.T. Horowitz and R.C. Myers, Black holes radiate mainly on the brane, Phys. Rev. Lett. 85 (2000) 499 hep-th/0003118.

[6] E. Jung and D.K. Park, Absorption and emission spectra of an higher-dimensional Reissner-Nordstróm black hole, Nucl. Phys. B 717 (2005) 272 hep-th/0502002;

N.G. Sanchez, Absorption and emission spectra of a schwarzschild black hole, Phys. Rev. D 18 (1978) 1030;

E. Jung and D.K. Park, Effect of scalar mass in the absorption and emission spectra of Schwarzschild black hole, Class. and Quant. Grav. 21 (2004) 3717 hep-th/0403251.

[7] A.S. Majumdar and N. Mukherjee, Braneworld black holes in cosmology and astrophysics, Int. J. Mod. Phys. D 14 (2005) 1095 astro-ph/0503473;

G. Kofinas, E. Papantonopoulos and V. Zamarias, Black hole solutions in braneworlds with induced gravity, Phys. Rev. D 66 (2002) 104028 [hep-th/0208207]; Black holes on the brane with induced gravity, Astrophys. Space Sci. 283 (2003) 685;

A.N. Aliev and A.E. Gumrukcuoglu, Charged rotating black holes on a 3-brane, Phys. Rev. D 71 (2005) 104027 hep-th/0502223; 
S. Kar and S. Majumdar, Black hole geometries in noncommutative string theory, Int. J. Mod. Phys. A 21 (2006) 6087 hep-th/0510043]; Noncommutative D(3)-brane, black holes and attractor mechanism, Phys. Rev. D 74 (2006) 066003 hep-th/0606026;

S. Kar, Tunneling between de Sitter and anti de Sitter black holes in a noncommutative D3-brane formalism, Phys. Rev. D 74 (2006) 126002 hep-th/0607029.

[8] V.P. Frolov and D. Stojkovic, Black hole as a point radiator and recoil effect on the brane world, Phys. Rev. Lett. 89 (2002) 151302 hep-th/0208102]; Black hole radiation in the brane world and recoil effect, Phys. Rev. D 66 (2002) 084002 hep-th/0206046;

D. Ida, K. Oda and S.C. Park, Rotating black holes at future colliders: greybody factors for brane fields, Phys. Rev. D 67 (2003) 064025 hep-th/0212108;

D. Stojkovic, Distinguishing between the small ADD and RS black holes in accelerators, Phys. Rev. Lett. 94 (2005) 011603 hep-ph/0409124;

E. Jung, S. Kim and D.K. Park, Condition for superradiance in higher-dimensional rotating black holes, Phys. Lett. B 615 (2005) 273 hep-th/0503163; Condition for the superradiance modes in higher-dimensional rotating black holes with multiple angular momentum parameters, Phys. Lett. B 619 (2005) 347 hep-th/0504139;

G. Duffy, C. Harris, P. Kanti and E. Winstanley, Brane decay of a $(4+n)$-dimensional rotating black hole: spin-0 particles, JHEP 09 (2005) 049 hep-th/0507274;

E. Jung and D.K. Park, Bulk versus brane in the absorption and emission: $5 D$ rotating black hole case, Nucl. Phys. B 731 (2005) 171 hep-th/0506204;

M. Casals, P. Kanti and E. Winstanley, Brane decay of a $(4+n)$-dimensional rotating black hole. II: spin-1 particles, JHEP 02 (2006) 051 hep-th/0511163;

A.S. Cornell, W. Naylor and M. Sasaki, Graviton emission from a higher-dimensional black hole, JHEP 02 (2006) 012 hep-th/0510009.

[9] D.K. Park, Hawking radiation of the brane-localized graviton from a $(4+n)$-dimensional black hole, Class. and Quant. Grav. 23 (2006) 4101 hep-th/0512021;

E. Jung and D.K. Park, Bulk versus brane in the Hawking radiation of graviton: black holes radiate mainly into the bulk when $n \geq 3$, hep-th/0612043;

V. Cardoso, M. Cavaglia and L. Gualtieri, Black hole particle emission in higher-dimensional spacetimes, Phys. Rev. Lett. 96 (2006) 071301 hep-th/0512002]; Hawking emission of gravitons in higher dimensions: non-rotating black holes, JHEP 02 (2006) 021 hep-th/0512116.

[10] D.-C. Dai, N. Kaloper, G.D. Starkman and D. Stojkovic, Evaporation of a black hole off of a tense brane, Phys. Rev. D 75 (2007) 024043 hep-th/0611184.

[11] L.-h. Liu, B. Wang and G.-h. Yang, The energy absorption problem of a brane-world black hole, Phys. Rev. D 76 (2007) 064014 hep-th/0701166.

[12] S. Creek, O. Efthimiou, P. Kanti and K. Tamvakis, Greybody factors for brane scalar fields in a rotating black-hole background, Phys. Rev. D 75 (2007) 084043 hep-th/0701288].

[13] S. Chen, B. Wang and R.-K. Su, Hawking radiation in a rotating Kaluza-Klein black hole with squashed horizons, Phys. Rev. D 77 (2008) 024039 arXiv:0710.3240.

[14] S. Creek, O. Efthimiou, P. Kanti and K. Tamvakis, Scalar emission in the bulk in a rotating black hole background, Phys. Lett. B 656 (2007) 102 arXiv:0709.0241; Greybody factors in a rotating black-hole background-II: fermions and gauge bosons, Phys. Rev. D 76 (2007)] 104013 arXiv:0707.1768. 
[15] J.-y. Shen, B. Wang and R.-K. Su, The signals from the brane-world black hole, Phys. Rev. D $\mathbf{7 4}$ (2006) 044036 hep-th/0607034.

[16] E. Abdalla, B. Cuadros-Melgar, A.B. Pavan and C. Molina, Stability and thermodynamics of brane black holes, Nucl. Phys. B 752 (2006) 40 gr-qc/0604033.

[17] S. Chen, B. Wang and R.-K. Su, Wave dynamics of a six-dimensional black hole localized on a tensional three-brane, Phys. Lett. B 647 (2007) 282 hep-th/0701209.

[18] P. Kanti, R.A. Konoplya and A. Zhidenko, Quasi-normal modes of brane-localised standard model fields. II: kerr black holes, Phys. Rev. D 74 (2006) 064008 gr-qc/0607048;

P. Kanti and R.A. Konoplya, Quasi-normal modes of brane-localised standard model fields, Phys. Rev. D 73 (2006) 044002 hep-th/0512257;

D.K. Park, Asymptotic quasinormal frequencies of brane-localized black hole, Phys. Lett. $\mathrm{B}$ 633 (2006) 613 hep-th/0511159.

[19] U.A. al Binni and G. Siopsis, Quasi-normal modes of a black hole localized on a codimension-two brane of finite tension, Phys. Rev. D 76 (2007) 104031 arXiv:0708.3363; H.T. Cho, A.S. Cornell, J. Doukas and W. Naylor, Fermion excitations of a tense brane black hole, arXiv:0710.5267; Bulk dominated fermion emission on a Schwarzschild background, arXiv:0709.1661.

[20] N. Kaloper and D. Kiley, Exact black holes and gravitational shockwaves on codimension-2 branes, JHEP 03 (2006) 077 hep-th/0601110.

[21] D. Kiley, Rotating black holes on codimension-2 branes, Phys. Rev. D 76 (2007) 126002 arXiv:0708.1016.

[22] T. Kobayashi, M. Nozawa and Y.-i. Takamizu, Bulk scalar emission from a rotating black hole pierced by a tense brane, arXiv:0711.1395. 